\title{
Optimized rapeseed oils rich in endogenous micronutrients ameliorate risk factors of atherosclerosis in high fat diet fed rats
}

Jiqu Xu' ${ }^{1,2}$, Congcong Ma ${ }^{1,2}$, Ling Han ${ }^{1,2}$, Hui Gao ${ }^{3}$, Qi Zhou ${ }^{1,2}$, Mei Yang ${ }^{1,2}$, Chang Chen ${ }^{4,5}$, Qianchun Deng ${ }^{1,2}$, Qingde Huang ${ }^{1,2}$ and Fenghong Huang ${ }^{1,2^{*}}$

\begin{abstract}
Background: Micronutrients in rapeseed such as polyphenols, tocopherols, phytosterols and phospholipids in rapeseed exert potential benefit to atherosclerosis. Some part of these healthy components substantially lost during the conventional refining processing. Thus some new processing technologies have been developed to produce various endogenous micronutrient-enriched optimized rapeseed oils. The aim of this study is to assess whether optimized rapeseed oils have positive effects on the atherosclerosis risk factors in rats fed a high-fat diet.
\end{abstract}

Methods: Rats received experiment diets containing 20\% fat and refined rapeseed oil or optimized rapeseed oils obtained with various processing technologies as lipid source. After 10 weeks of treatment, plasma was assayed for oxidative stress, lipid profiles and imflammation.

Results: Micronutrients enhancement in optimized rapeseed oils significantly reduced plasma oxidative stress, as evaluated by the significant elevation in the activities of CAT and GPx as well as the level of GSH, and the significant decline in lipid peroxidation. Optimized rapeseed oil with the highest micronutrient contents obtained by microwave pretreatment-cold pressing reduced the levels of TG, TC and LDL-C as well as IL-6 and CRP in plasma.

Conclusions: These results suggest that optimized rapeseed oils may contribute to prevent atherogenesis and make them very promising functional food in cardiovascular health promotion.

Keywords: Optimized rapeseed oils, Micronutrients, Atherosclerosis, Oxidant stress, Plasma lipids, Inflammation

\section{Introduction}

Cardiovascular disease (CVD) is the leading cause of premature death in most developed and developing countries and it is also an increasingly important source of disability and contributes in large part to the escalating costs of health care. Atherosclerosis, a manifestation of the pathophysiology underlying CVD, constitutes the single most important contributor to this growing burden of this disease. There are definitive evidences to show that oxidant stress [1], lipid abnormalities [2] as

\footnotetext{
* Correspondence: fhhuang@foxmail.com

'Department of Product Processing and Nutriology, Oil Crops Research Institute, Chinese Academy of Agricultural Sciences, 2 Xudong Second Road, Wuhan 430062, P.R. China

${ }^{2}$ Hubei Key Laboratory of Lipid Chemistry and Nutrition, 2 Xudong Second Road, Wuhan 430062, P.R. China

Full list of author information is available at the end of the article
}

well as chronic inflammation [3] have a crucial involvement in both the initiation and the progression of atherosclerosis.

Rapeseed is a major oilseed crop in China and many other countries. It contains high-quality oil which is one of the most common and cheapest vegetable oils for human diet. Rapeseed oil has the exceptionally low amount of saturated fatty acids in all commodity edible oils and high level of monounsaturated fatty acids [4]. Besides, this kind of plant oil is also naturally rich in $\alpha$-linolenic acid and linoleic acid whose ratio are the closest to the optimum to meet the basic requirements of essential fatty acids in the body [5]. In addition to triacylglycerols, rapeseed also contains many healthy bioactive compounds such as phenolic compounds, tocopherols and phytosterols and these endogenous micronutrients have 
been reported to possess many health benefits or desirable physiological effects in cardiovascular system. For example, by their abilities to scavenge reactive oxygen species (ROS) directly or form complexes with prooxidant metals, these micronutrients possess a potent antioxidant activity and the various bioavailable antioxidants present in rapeseed oil work in concert to upgrade the complex antioxidant network which increase antioxidant capacity higher than that provided by each separate compound [6-8]. Phytosterols have been reported to inhibit cholesterol absorption and thus reduce circulating levels of total (TC) and low density lipoprotein cholesterol (LDL-C) [9]. Previous studies have also shown an independent effect of phenolics improving plasma lipid profiles $[10,11]$. Also, all these compounds are known to have antiinflammatory effects [11-13]. The beneficial effects of these inherent micronutrients might contribute to prevent the initiation and development of atherosclerosis. However, the conventional industrial processes (extraction and refining) lead to substantial losses of these cardiovascular protective micronutrients. In order to improve on the desirable components retention and then to develop new healthy oils, some new processing technologies have been developed recently. The aim of this study is to determine the effects of the various endogenous micronutrient-enriched optimized rapeseed oils on atherosclerosis risk factors in rats fed a high-fat diet.

\section{Materials and methods}

\section{Oils preparation}

Rapeseed oils were produced with different technical procedures. The refined rapeseed oil (RRO) was prepared with conventional extraction and refining processing technology. Other three rapeseed oil processing technologies were applied to obtain high levels of endogenous micronutrients: cold pressing $(\mathrm{CP})$, dehulling-cold pressing (DCP) and microwave pretreatment-cold pressing (MPCP). The fatty acid compositions and endogenous micronutrients contents in different rapeseed oil were shown in Tables 1 and 2, respectively.

\section{Animals and diets}

Forty male Wister rats, initially weighing $150-170 \mathrm{~g}$, were obtained from Vital River Laboratory Animal Center

Table 1 Fatty acid compositions in different rapeseed oils

\begin{tabular}{lcccc}
\hline Fatty acid (wt.\%) & RRO & CP & DCP & MPCP \\
\hline Palmitic acid (C16:0) & 2.413 & 2.5 & 2.485 & 2.44 \\
Stearic acid (C18:0) & 3.667 & 3.769 & 3.846 & 3.548 \\
Oleic acid (C18:1) & 66.19 & 66.075 & 66.287 & 66.561 \\
Linoleic acid (C18:2) & 16.822 & 16.798 & 17.088 & 16.447 \\
Linolenic acid (C18:3) & 9.146 & 9.281 & 8.463 & 9.431 \\
\hline
\end{tabular}

Table 2 Endogenous micronutrients contents in different rapeseed oils

\begin{tabular}{lcccc}
\hline $\mathbf{m g} / \mathbf{k g}$ oil & RRO & CP & DCP & MPCP \\
\hline Phenols (in eq., sinapic acid) & 8 & 34 & 43 & 645 \\
Of which canolol & ND* & 107 & 167 & 816 \\
Phytosterols & 9592 & 9902 & 8834 & 11027 \\
Tocopherol & 461 & 541 & 594 & 600 \\
Phospholipids & 22 & 600 & 590 & 1330 \\
\hline
\end{tabular}

${ }^{*} \mathrm{ND}$, not detected.

(Beijing, China). The rats were housed individually and maintained at a controlled ambient temperature $\left(24 \pm 1^{\circ} \mathrm{C}\right)$ under diurnal conditions (light-dark: 08:00-20:00) with access to laboratory chow and tap water ad libitum. After the rats were acclimated for 1 week, animals were randomly divided into four groups of 10 rats each, consisting of RRO, CP, DCP and MPCP groups. The high-fat diet contained $20 \%$ casein, $35 \%$ maize starch, $15 \%$ glucose, $5 \%$ cellulose, $3.5 \%$ mineral mixture (AIN-93 M), 1\% vitamin mixture (AIN-93 M), 0.2\% choline bitartrate, 0.3\% DLmethionine and $20 \%$ fat. The fat in the diet was provided by different rapeseed oils mentioned above. All animals were weighed twice a week and food intake was measured weekly. The animals were cared for in accordance with the Guiding Principles in the Care and Use of Animals. The experiment was approved by the Oil Crops Research Institute Council on Animal Care Committee, Chinese Academy of Agricultural Sciences.

\section{Blood processing}

After 10 weeks of treatment, rats were fasted for 16 hours and then killed under anaesthesia, blood was collected in the presence of sodium heparin from the heart immediately. Blood samples were centrifuged at $1500 \mathrm{~g}$ $\left(10 \mathrm{~min}, 4^{\circ} \mathrm{C}\right)$ and the plasma was stored at $-80^{\circ} \mathrm{C}$ until analysis.

\section{Plasma lipids analysis}

The plasma triglyeride (TG), TC, LDL-C and highdensity lipoprotein cholesterol (HDL-C) levels were determined with commercial kits (Wako, Japan) by Hitachi 7020 full-automatic biochemical analyzer (Japan).

Assay of plasma antioxidant capacity and lipid peroxidation Superoxide dismutases (SOD) activity was estimated according to the method of Kono [14]. Catalase (CAT) activity was measured basing on the method of Goth [15]. Glutathione peroxidase (GPx) activity was determined by the method of Sazuka [16]. The glutathione (GSH) content was assayed by the method of Moron [17]. Thiobarbituric acid reactive substances (TBARS) level was assayed by the method of Buege [18]. The detection procedure of these 
enzymes activities has been described in detail in our preceding report [19].

\section{Assay of plasma inflammatory markers}

The plasma interleukin 6 (IL-6) and C-reactive protein (CRP) levels were measured by means of commercially available Rat CRP ELISA kit (Abcam, Cambridge, MA) and Rat IL-6 ELISA kit (Abcam, Cambridge, MA), respectively. All the procedures and conditions were consistent with the instructions of these kits.

\section{Statistical analyses}

Values are presented as mean \pm SEM (standard error of the mean). The data were analyzed by one-way ANOVA, followed by the Fisher PLSD post hoc test if the overall differences were significant $(p<0.05)$. All statistical analyses were performed using SPSS 13.0 statistical software (SPSS Inc., Chicago, IL) and a difference was considered significant when $p<0.05$.

\section{Results}

\section{Plasma lipids}

As can be seen in Figure 1, the plasma TG levels showed significant decreases in $\mathrm{CP}$ and MPCP groups than RRO group ( $p<0.05$ and 0.01 , respectively). Although compatible HDL-C levels in plasma were observed among all groups $(p>0.05)$, animals in MPCP group exhibited marked decline in TC and LDL-C levels as well as the
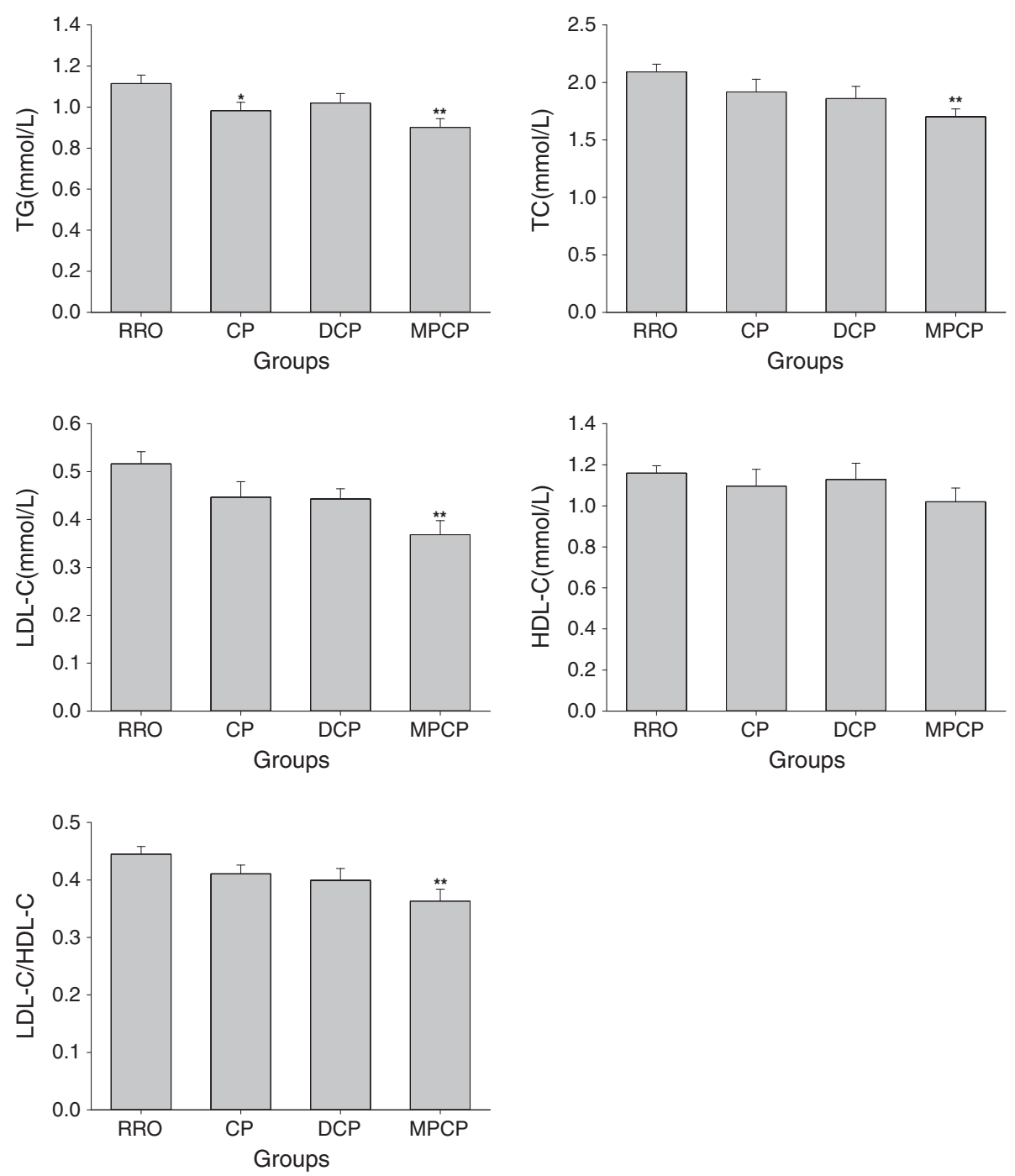

Figure 1 Effects of optimized rapeseed oils on various plasma lipid parameters (TG, TC, LDL-C and HDL-C) and on the ratios of plasma LDL-C/HDL-C of rats fed a high-fat diet. RRO: the refined rapeseed oil group; CP: cold pressing rapeseed oil group; DCP: dehulling-cold pressing rapeseed oil group; MPCP: microwave pretreatment-cold pressing rapeseed oil group. Bars represent the mean \pm SEM from 10 animals in each group. ${ }^{*} p<0.05$ and ${ }^{* *} p<0.01$ compared to the RRO group. 
ratio of LDL-C/HDL-C in plasma compared with those in the RRO group $(p<0.01)$.

\section{Plasma antioxidative capacity and lipid peroxidation}

As shown in Figure 2, there were no significant differences in plasma SOD activities among various rapeseed oil groups $(p>0.05)$. Animals in CP, DCP and MPCP groups displayed significantly higher GPx activities $(p<0.05,0.05$ and 0.01 , respectively) and rats in DCP and MPCP groups had marked enhancement of CAT activities $(p<0.05$ and 0.01 , respectively) when compared with their counterparts in RRO group. Besides, plasma GSH levels were also found to be elevated in CP and MPCP groups as compared to that in RRO group ( $p<0.05$ and 0.01 , respectively). When plasma TBARS were evaluated as the marker of lipid peroxidation, animals in MPCP group revealed markedly lower TBARS levels than that in RRO group $(p<0.01)$.

\section{Plasma inflammatory}

When the plasma IL-6 and CRP levels were examined as the systemic markers for inflammation, both of them were affected by the micronutrient contents of experimental oils which have been shown in Figure 3. The plasma levels of IL-6 in DCP and MPCP groups $(p<0.01)$ as well as CRP in $\mathrm{CP}$ and MPCP groups ( $p<0.05$ and 0.01 , respectively) were significantly lower than those in $\mathrm{RRO}$ group.

\section{Discussion}

Although considerable progress has been made in the treatment of CVD with drug therapy over the past decades,
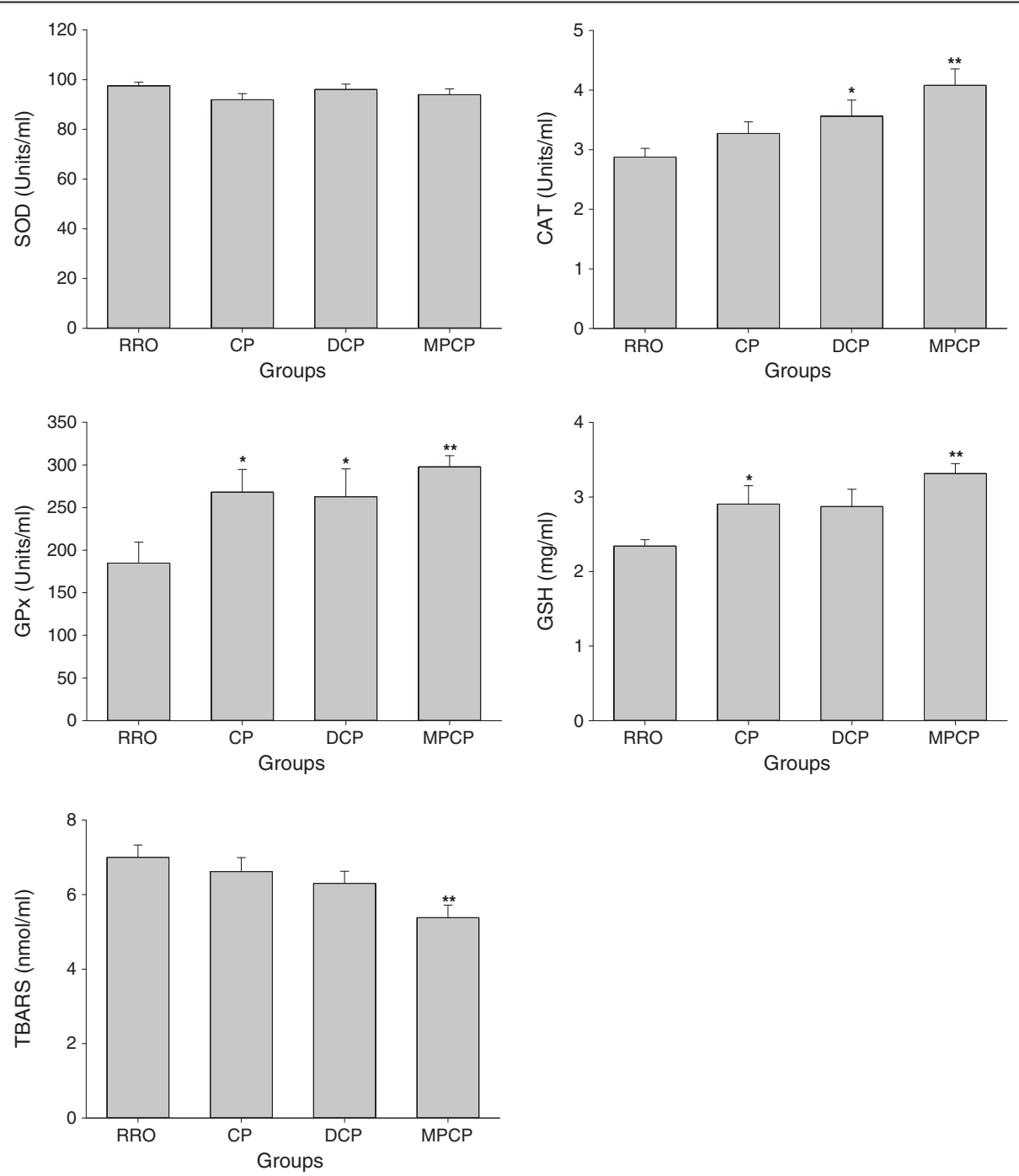

Figure 2 Effects of optimized rapeseed oils on antioxidant enzymes (SOD, CAT and GPx) activities, GSH and TBARS contents in plasma of rats fed a high-fat diet. RRO: the refined rapeseed oil group; CP: cold pressing rapeseed oil group; DCP: dehulling-cold pressing rapeseed oil group; MPCP: microwave pretreatment-cold pressing rapeseed oil group. Bars represent the mean \pm SEM from 10 animals in each group. ${ }^{*} p<0.05$ and ${ }^{* *} p<0.01$ compared to the RRO group. 

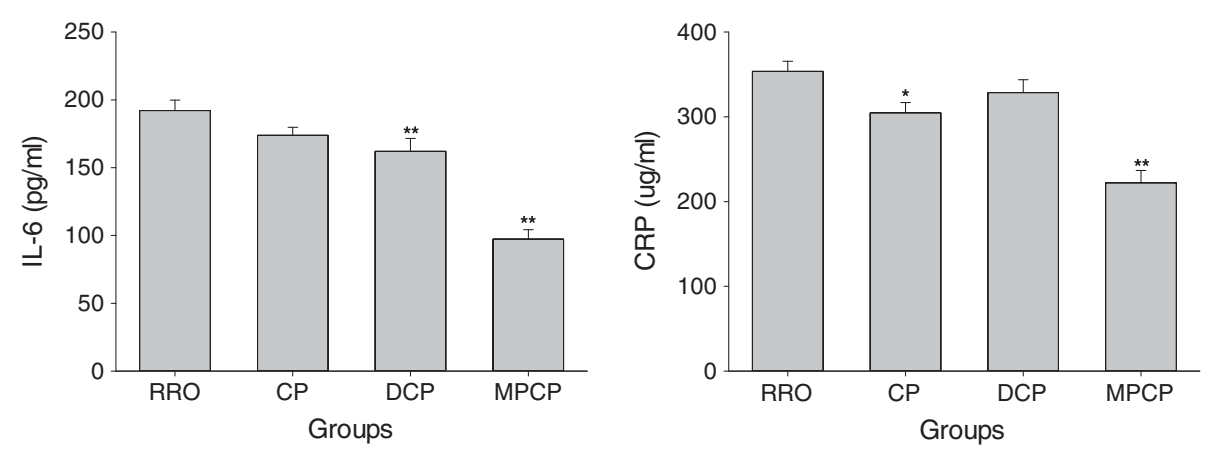

Figure 3 Effects of optimized rapeseed oils on IL-6 and CRP levels in plasma of rats fed a high-fat diet. RRO: the refined rapeseed oil group; CP: cold pressing rapeseed oil group; DCP: dehulling-cold pressing rapeseed oil group; MPCP: microwave pretreatment-cold pressing rapeseed oil group. Bars represent the mean \pm SEM from 10 animals in each group. ${ }^{*} p<0.05$ and ${ }^{* *} p<0.01$ compared to the RRO group.

this disease still remains the dominant epidemic in the world. It is widely understood that high fat diets, especially saturated fat, are implicated in the onset and development of CVD. High fat intake leads to plasma lipid abnormalities [20], including hypertriglyceridemia and hypercholesterolemia, oxidant stress [20] as well as low-grade system inflammation [21], which are major risk factors for CVD development [1-3].

After most of the erucic acid is removed by genetic engineering, the production and consumption of rapeseed oil leaped into prominence. Rapeseed oil ingestion has shown beneficial effects for CVD prevention. For example, replacing dairy fat with rapeseed oil can cause rapid and clinically relevant reductions in serum TG, TC, LDL-C and the ratio of LDL-C/HDL-C for hyperlipidaemic individuals [22]. The lipid-lowering efficacy of refined rapeseed oil is related to the sufficient amounts of alpha-linolenic acid (ALA) [23,24]. ALA has been reported to suppress the expression and activities of many hepatic fatty acid syntheses such as fatty acid synthase (FAS), malic enzyme and glucose 6-phosphate dehydrogenase $[25,26]$. On the other hand, ALA also sharply upregulates hepatic peroxisomal and mitochondrial fatty acid oxidation rate by increasing the expression and activities of a series of fatty acid oxidation enzymes [26,27]. In addition, ALA has been shown to enhance hepatic LDL-receptor expression and cholesterol catabolism/ output [28].

Processing technologies decisively and directly have an influence on the quantity of micronutrients in oils. In this study, all optimized rapeseed oils obtained with new processing technologies contain more micronutrients and it is noteworthy that MPCP made the greatest micronutrient retention in oil. These bioactive compounds act synergistically with each other and exert more potent biological effects in combination than as single nutrients. As predicted, the optimized rapeseed oils provided additional hypolipidaemic effects than refined rapeseed oil and these endogenous micronutrients should responsible for the apparent boon. Phenols have been shown to reduce plasma TG, TC and LDL-C by altering hepatic triglyceride assembly and secretion, cholesterol absorption and the processing of lipoproteins in plasma [11]. Phospholipids, another dramatically increased cardiovascular protective micronutrients in optimized rapeseed oils, have been consistently demonstrated to have the ability to reduce plasma triglyceride and cholesterol [29]. Phytosterols have a similar chemical structure with cholesterol but themselves are absorbed only in trace amounts [30], thus they inhibit cholesterol absorption including recirculating endogenous biliary cholesterol which is a key step in cholesterol elimination [30]. In addition, the hypolipidaemic effects of the phytosterols were also associated with the down-regulation of hepatic acyl CoA:cholesterol acytransferase activity [31] and the increasing LDL receptor expression [32].

The imbalance between the cellular free radical formation and the antioxidant defense leads to oxidative stress. The relative excessive production of free radicals can attack and denature many different cellular components, including lipids, proteins and DNA, which initiates the processes of atherogenesis through cell dysfunction [33]. In fact, oxidative stress is the unifying mechanism for many CVD risk factors [34]. For example, native LDL becomes oxidized in response to free radicals leading to the formation of oxidized LDL [12,34] which plays an important role in the genesis and progression of atherosclerosis [12]. However, the deleterious effects of oxidative stress can be prevented by enzymatic and nonenzymatic antioxidant defense mechanism. In mammals, the most important antioxidant enzymes include SOD which converts superoxide to hydrogen peroxide, GPx and CAT which are responsible for converting hydrogen peroxide to water [35]. GSH is a very important nonenzymatic antioxidant, which can react directly with free radical or act an electron donor in the reduction of 
peroxides catalyzed by GPx [36]. The main phenolic compounds in rapeseed oil are sinapic acid and its derivatives [37]. Sinapic acid can efficiently scavenge free radicals through an electron donation mechanism [38]. Canolol (4-Vinylsyringol) is one of derivatives of sinapic acid and the mainly phenols in optimized rapeseed oils, which is more efficient as an radical scavenger than many other antioxidants, including $\alpha$-tocopherol, vita$\min C, \beta$-carotene, rutin, and quercetin [39]. Tocopherol is also well known to acts as a powerful antioxidant by breaking chain reactions propagated by free radicals. Besides, the increased micronutrient contents in optimized rapeseed oils were accompanied with the marked increase of the plasma antioxidant enzymes CAT and GPx activities as well as GSH contents in the present study. All of these indicated that the micronutrients in optimized rapeseed oils had abilities to enhance the antioxidant defense system. As results, MPCP oil which possesses the highest micronutrients contents had appreciable ability to reduce plasma lipid peroxidation level. Similar positive effects were also observed by elevating micronutrients levels in oils with different manners [19,40-42].

Recent advances in both the basic and clinical science have recognized the critical role of inflammation in all stages of atherosclerosis [43-45]. Various proinflammatory risk factors (oxidized LDL, infectious agents, etc.) can trigger the production of proinflammatory cytokines which contribute to development and progression of atherosclerosis. IL-6 and CRP are two of the most important proinflammatory cytokines, and both of which have been served as inflammatory markers for evaluation of atherosclerotic risk [12,46-48]. The enhancement of micronutrients in optimized rapeseed oils in the present study tended to reduce the levels of plasma IL-6 and CRP, and further, significant decreases of both inflammation markers were observed with the consumption of MPCP oil. Both sinapic acid and canolol have been reported to suppresses the expression of many proinflammatory mediators including inducible nitric oxide synthase, cyclooxygenase-2, tumor necrosis factor- $\alpha$, and interleukin- $1 \beta$ via NF- $\mathrm{kB}$ inactivation and thus exert their anti-inflammatory effects [49,50]. Tocopherol also exerts anti-inflammatory properties by reducing many biomarkers of inflammation in atherosclerosis [12]. Besides, since free radicals mediate many signaling pathways which underlie vascular inflammation in atherogenesis [51], all these antioxidants exert actions as inflammation preventive agents via antioxidation.

In conclusion, the optimized rapeseed oils produced by some new processing technologies have the abilities to improve plasma oxidative stress, lipid profile and inflammation in high fat diet fed rats and these positive effects were more pronounced for MPCC oil due to the most abundant inherent micronutrients in this oil. These results suggested that the optimized rapeseed oils rich in endogenous micronutrients might contribute to prevent atherogenesis and make them very promising functional food in cardiovascular health promotion.

\section{Competing interests}

The authors declare that they have no competing interests.

\section{Authors' contributions}

The author JX designed and wrote a first draft of the paper. CM, LH, HGand CC carried out all the experiments. QZ, MY and QD prepared and analyzed oils. QH performed the data analysis and created the figures. FH contributed to the design of the study, reviewed the manuscript and contributed to the final version. All authors contributed to and have approved the final manuscript.

\section{Acknowledgments}

This work was supported by National Natural Science Foundation of China (NSFC-31271856) and the earmarked fund for Modern Agro-industry Technology Research System, China.

\section{Author details}

'Department of Product Processing and Nutriology, Oil Crops Research Institute, Chinese Academy of Agricultural Sciences, 2 Xudong Second Road, Wuhan 430062, P.R. China. ${ }^{2}$ Hubei Key Laboratory of Lipid Chemistry and Nutrition, 2 Xudong Second Road, Wuhan 430062, P.R. China. ${ }^{3}$ Department of Nutrition and Food Hygiene, School of Public Health, Tongji Medical College, Huazhong University of Science and Technology, 13 Hangkong Road, Wuhan 430030, P.R. China. ${ }^{4}$ Department of Gastroenterology, The First People's Hospital of Yichang, The People's Hospital of China Three Gorges University, 2 Jiefang Road, Yichang 443000, P.R. China. ${ }^{5}$ Department of Gastroenterology, The People's Hospital of China Three Gorges University, 2 Jiefang Road, Yichang 443000, P.R. China.

Received: 18 August 2014 Accepted: 21 October 2014 Published: 30 October 2014

\section{References}

1. Yokoyama M: Oxidant stress and atherosclerosis. Curr Opin Pharmacol 2004, 4(2):110-115.

2. Castelli WP, Garrison RJ, Wilson PW, Abbott RD, Kalousdian S, Kannel WB: Incidence of coronary heart disease and lipoprotein cholesterol levels. Framingham Stud JAMA 1986, 256(20):2835-2838.

3. Hansson GK: Inflammation, atherosclerosis, and coronary artery disease. N Engl J Med 2005, 352(16):1685-1695.

4. Johnson GH, Keast DR, Kris-Etherton PM: Dietary modeling shows that the substitution of canola oil for fats commonly used in the United States would increase compliance with dietary recommendations for fatty acids. J Am Diet Assoc 2007, 107(10):1726-1734.

5. Seppanen-Laakso T, Laakso I, Lehtimaki T, Rontu R, Moilanen E, Solakivi T, Seppo L, Vanhanen H, Kiviranta K, Hiltunen R: Elevated plasma fibrinogen caused by inadequate alpha-linolenic acid intake can be reduced by replacing fat with canola-type rapeseed oil. Prostag Leukotr Essent Fatty Acids 2010, 83(1):45-54.

6. Chen CY, Milbury PE, Lapsley K, Blumberg JB: Flavonoids from almond skins are bioavailable and act synergistically with vitamins $C$ and $E$ to enhance hamster and human LDL resistance to oxidation. J Nutr 2005, 135(6):1366-1373.

7. Vivancos M, Moreno JJ: Effect of resveratrol, tyrosol and beta-sitosterol on oxidised low-density lipoprotein-stimulated oxidative stress, arachidonic acid release and prostaglandin E2 synthesis by RAW 264.7 macrophages. Br J Nutr 2008, 99(6):1199-1207.

8. Devaraj S, Leonard S, Traber MG, Jialal I: Gamma-tocopherol supplementation alone and in combination with alpha-tocopherol alters biomarkers of oxidative stress and inflammation in subjects with metabolic syndrome. Free Radic Biol Med 2008, 44(6):1203-1208.

9. Wester I: Cholesterol-lowering effect of plant sterols. Eur J Lipid Sci Technol 2000, 102(1):37-44

10. Covas MI, Nyyssonen K, Poulsen HE, Kaikkonen J, Zunft HJ, Kiesewetter $\mathrm{H}$, Gaddi A, de la Torre R, Mursu J, Baumler H, Nascetti S, Salonen JT, Fito M, 
Virtanen J, Marrugat J: The effect of polyphenols in olive oil on heart disease risk factors: a randomized trial. Ann Intern Med 2006, 145(5):333-341

11. Zern TL, Fernandez ML: Cardioprotective effects of dietary polyphenols. J Nutr 2005, 135(10):2291-2294

12. Singh $U$, Devaraj $S$, Jialal I: Vitamin E, oxidative stress, and inflammation. Annu Rev Nutr 2005, 25:151-174.

13. Bouic PJ: The role of phytosterols and phytosterolins in immune modulation: a review of the past 10 years. Curr Opin Clin Nutr Metab Care 2001, 4(6):471-475

14. Kono Y: Generation of superoxide radical during autoxidation of hydroxylamine and an assay for superoxide dismutase. Arch Biochem Biophys 1978, 186(1):189-195.

15. Goth L: A simple method for determination of serum catalase activity and revision of reference range. Clin Chim Acta 1991, 196(2-3):143-151.

16. Sazuka $Y$, Tanizawa $H$, Takino $Y$ : Effect of adriamycin on the activities of superoxide dismutase, glutathione peroxidase and catalase in tissues of mice. Jpn J Cancer Res 1989, 80(1):89-94.

17. Moron MS, Depierre JW, Mannervik B: Levels of glutathione, glutathione reductase and glutathione S-transferase activities in rat lung and liver. Biochim Biophys Acta 1979, 582(1):67-78

18. Buege JA, Aust SD: Microsomal lipid peroxidation. Methods Enzymol 1978 52:302-310

19. Xu J, Zhou X, Deng Q, Huang Q, Yang J, Huang F: Rapeseed oil fortified with micronutrients reduces atherosclerosis risk factors in rats fed a high-fat diet. Lipids Health Dis 2011, 10:96.

20. Yang RL, Li W, Shi YH, Le GW: Lipoic acid prevents high-fat diet-induced dyslipidemia and oxidative stress: a microarray analysis. Nutrition 2008 24(6):582-588

21. Terra X, Montagut G, Bustos M, Llopiz N, Ardevol A, Blade C, FernandezLarrea J, Pujadas G, Salvado J, Arola L, Blay M: Grape-seed procyanidins prevent low-grade inflammation by modulating cytokine expression in rats fed a high-fat diet. J Nutr Biochem 2009, 20(3):210-218.

22. Iggman D, Gustafsson IB, Berglund L, Vessby B, Marckmann P, Riserus U: Replacing dairy fat with rapeseed oil causes rapid improvement of hyperlipidaemia: a randomized controlled study. J Intern Med 2011, 270(4):356-364.

23. Riediger ND, Othman R, Fitz E, Pierce GN, Suh M, Moghadasian MH: Low n-6:n-3 fatty acid ratio, with fish- or flaxseed oil, in a high fat diet improves plasma lipids and beneficially alters tissue fatty acid composition in mice. Eur J Nutr 2008, 47(3):153-160.

24. Vijaimohan K, Jainu M, Sabitha KE, Subramaniyam S, Anandhan C, Shyamala Devi CS: Beneficial effects of alpha linolenic acid rich flaxseed oil on growth performance and hepatic cholesterol metabolism in high fat diet fed rats. Life Sci 2006, 79(5):448-454.

25. Kim HK, Choi S, Choi H: Suppression of hepatic fatty acid synthase by feeding alpha-linolenic acid rich perilla oil lowers plasma triacylglycerol level in rats. J Nutr Biochem 2004, 15(8):485-492.

26. Ide T, Kobayashi H, Ashakumary L, Rouyer IA, Takahashi Y, Aoyama T, Hashimoto T, Mizugaki M: Comparative effects of perilla and fish oils on the activity and gene expression of fatty acid oxidation enzymes in rat liver. Biochim Biophys Acta 2000, 1485(1):23-35.

27. Kabir $Y$, Ide $T$ : Activity of hepatic fatty acid oxidation enzymes in rats fed alpha-linolenic acid. Biochim Biophys Acta 1996, 1304(2):105-119.

28. Tzang BS, Yang SF, Fu SG, Yang HC, Sun HL, Chen YC: Effects of dietary flaxseed oil on cholesterol metabolism of hamsters. Food Chem 2009, 114(4):1450-1455

29. Cohn JS, Wat E, Kamili A, Tandy S: Dietary phospholipids, hepatic lipid metabolism and cardiovascular disease. Curr Opin Lipidol 2008, 19(3):257-262.

30. Ostlund RE Jr: Phytosterols in human nutrition. Annu Rev Nutr 2002, 22:533-549.

31. Shin J, Kim YJ, Choi MS, Woo DH, Park T: Phytosterols and lecithin do not have an additive effect in lowering plasma and hepatic cholesterol levels in diet-induced hypercholesterolemic rats. Biofactors 2004, 22(1-4):173-175.

32. Plat J, Mensink RP: Effects of plant stanol esters on LDL receptor protein expression and on LDL receptor and HMG-CoA reductase mRNA expression in mononuclear blood cells of healthy men and women. FASEB J 2002, 16(2):258-260.
33. Harrison D, Griendling KK, Landmesser U, Hornig B, Drexler H: Role of oxidative stress in atherosclerosis. Am J Cardio/ 2003, 91(3A):7A-11A.

34. Madamanchi NR, Vendrov A, Runge MS: Oxidative stress and vascular disease. Arterioscler Thromb Vac Biol 2005, 25(1):29-38.

35. Formigari $A$, Irato $P$, Santon $A$ : Zinc, antioxidant systems and metallothionein in metal mediated-apoptosis: biochemical and cytochemical aspects. Comp Biochem Physiol C Toxicol Pharmacol 2007, 146(4):443-459.

36. Dringen R: Metabolism and functions of glutathione in brain. Prog Neurobiol 2000, 62(6):649-671.

37. Harbaum-Piayda B, Oehlke K, Sönnichsen FD, Zacchi P, Eggers R, Schwarz K: New polyphenolic compounds in commercial deodistillate and rapeseed oils. Food Chem 2010, 123(3):607-615.

38. Zou Y, Kim AR, Kim JE, Choi JS, Chung HY: Peroxynitrite scavenging activity of sinapic acid (3,5-dimethoxy-4-hydroxycinnamic acid) isolated from Brassica juncea. J Agric Food Chem 2002, 50(21):5884-5890.

39. Wakamatsu D, Morimura S, Sawa T, Kida K, Nakai C, Maeda H: Isolation, identification, and structure of a potent alkyl-peroxyl radical scavenger in crude canola oil, canolol. Biosci Biotechnol Biochem 2005, 69(8):1568-1574.

40. Xu J, Zhou X, Gao H, Chen C, Deng Q, Huang Q, Ma J, Wan Z, Yang JE, Huang F: Micronutrients-fortified rapeseed oil improves hepatic lipid accumulation and oxidative stress in rats fed a high-fat diet. Lipids Health Dis 2013, 12(1):28.

41. Attorri L, Di Biase A, Di Benedetto R, Rigato P, Di Virgilio A, Salvati S: Micronutrient-enriched rapeseed oils reduce cardiovascular disease risk factors in rats fed a high-fat diet. Atherosclerosis 2010, 213(2):422-428.

42. Di Benedetto R, Attorri L, Chiarotti F, Eusepi A, Di Biase A, Salvati S: Effect of micronutrient-enriched sunflower oils on plasma lipid profile and antioxidant status in high-fat-fed rats. J Agric Food Chem 2010, 58(9):5328-5333.

43. Libby P: Inflammation in atherosclerosis. Nature 2002, 420(6917):868-874

44. Willerson JT, Ridker PM: Inflammation as a cardiovascular risk factor. Circulation 2004, 109(21 Suppl 1):I12-II10.

45. Libby P, Ridker PM, Hansson GK, Leducq Transatlantic Network on A: Inflammation in atherosclerosis: from pathophysiology to practice. J Am Coll Cardiol 2009, 54(23):2129-2138.

46. Ridker PM, Rifai N, Rose L, Buring JE, Cook NR: Comparison of C-reactive protein and low-density lipoprotein cholesterol levels in the prediction of first cardiovascular events. N Engl J Med 2002, 347(20):1557-1565.

47. Tzoulaki I, Murray GD, Lee AJ, Rumley A, Lowe GD, Fowkes FG: C-reactive protein, interleukin- 6 , and soluble adhesion molecules as predictors of progressive peripheral atherosclerosis in the general population: Edinburgh Artery Study. Circulation 2005, 112(7):976-983.

48. Larsson PT, Hallerstam S, Rosfors S, Wallen NH: Circulating markers of inflammation are related to carotid artery atherosclerosis. Int Angio/ 2005, 24(1):43-51

49. Yun KJ, Koh DJ, Kim SH, Park SJ, Ryu JH, Kim DG, Lee JY, Lee KT: Anti-inflammatory effects of sinapic acid through the suppression of inducible nitric oxide synthase, cyclooxygase-2, and proinflammatory cytokines expressions via nuclear factor-kappaB inactivation. J Agric Food Chem 2008, 56(21):10265-10272.

50. Cao X, Tsukamoto T, Seki T, Tanaka H, Morimura S, Cao L, Mizoshita T, Ban H, Toyoda T, Maeda H, Tatematsu M: 4-Vinyl-2,6-dimethoxyphenol (canolol) suppresses oxidative stress and gastric carcinogenesis in Helicobacter pylori-infected carcinogen-treated Mongolian gerbils. Int J Cancer 2008 122(7):1445-1454.

51. Singh U, Jialal I: Oxidative stress and atherosclerosis. Pathophysiology 2006, 13(3):129-142.

doi:10.1186/1476-511X-13-166

Cite this article as: Xu et al:: Optimized rapeseed oils rich in endogenous micronutrients ameliorate risk factors of atherosclerosis in high fat diet fed rats. Lipids in Health and Disease 2014 13:166. 